\title{
Beneficial effects of LK-4, an analog of dextromethorphan on lipopolysaccharide-induced sepsis in rats
}

\author{
Shyi-Shiaw Jiau ${ }^{1}$, Pao-Yun Cheng ${ }^{2}$, Yen-Mei Lee ${ }^{2}$, Wen-Hsin Huang ${ }^{3}$ \\ Ya-Fang $\mathrm{Ko}^{2}$ \& Mao-Hsiung Yen ${ }^{2, *}$ \\ ${ }^{1}$ Division of Cardiology, Department of Medicine, Tri-Service General Hospital, National Defense Medical \\ Center, Taipei, Taiwan; ${ }^{2}$ Department of Pharmacology, National Defense Medical Center, P.O. Box $90048-$ \\ 504 Nei-Hu 114, Taipei, Taiwan; ${ }^{3}$ Department of Pharmacy, National Defense Medical Center, Taipei, \\ Taiwan
}

Received 16 June 2006; accepted 4 September 2006

(C) 2006 National Science Council, Taipei

Key words: dextromethorphan, reactive oxygen species, sepsis, TNF- $\alpha$, nitric oxide, circulatory failure

\begin{abstract}
Summary
Dextromethorphan (DM), an anti-tussive agent, has been claimed to have anti-inflammatory and immunomodulatory effects in vitro. In our preliminary screening test, LK-4, an analog of DM, can afford more protection against circulatory failure induced by LPS than that of DM. Thus, the aim of this study was to evaluate the effects of LK-4 on sepsis induced by intravenous (i.v.) administration of lipopolysaccharide (LPS; $10 \mathrm{mg} / \mathrm{kg}$ ) in anesthetized Wistar rats and survival rate by intraperitoneal administration of LPS $(70 \mathrm{mg} / \mathrm{kg}$ ) in conscious ICR mice. Results demonstrated that posttreatment with LK-4 (3 and $5 \mathrm{mg} /$ $\mathrm{kg}$, i.v.) significantly attenuated the deleterious hemodynamic changes (e.g., hypotension and tachycardia) in rats treated with LPS. Meanwhile, LK-4 $(3 \mathrm{mg} / \mathrm{kg})$ significantly inhibited the elevation of plasma tumor necrosis factor- $\alpha$, as well as values of GOT and GPT, and BUN and creatinine caused by LPS. The induction of inducible NO synthase and the overproduction of NO and superoxide anions by LPS were also reduced by LK-4. Moreover, infiltration of neutrophils into the lungs and liver of rats $8 \mathrm{~h}$ after treatment with LPS was also reduced by LK-4. Furthermore, LK-4 increased the survival rate of mice insulted by toxic dose of LPS. In conclusion, the beneficial effects of LK-4 on LPS-induced sepsis result from its antiinflammatory and anti-oxidant effects. Thus, LK-4 can be potentially used as a therapeutic agent for sepsis in the future.
\end{abstract}

\section{Introduction}

Sepsis is generally considered to be a systemic inflammatory disorder. It can be defined as a progressive failure of the circulation, clinically characterized by systemic hypotension, hyporeactiveness to vasoconstrictors, and subsequent organ perfusion and function abnormities followed by multiple organ failure [1]. Septic shock is a serious

*To whom correspondence should be addressed. Fax: + 886-287921704; E-mail: mhyen@mail.ndmctsgh.edu.tw clinical problem with high mortality [1, 2]. According to Bone et al. [1], the progression from septicemia to septic shock involves several steps. First, local inflammation may provoke the release of proinflammatory mediators, such as tumor necrosis factor (TNF) and interleukins to reduce tissue injury. Then, proinflammatory mediators cause migration of leukocytes, lymphocytes, and platelets to infected areas. Systemic pathologic changes include endothelial damage, increased microvascular permeability, platelet aggregation, local blood flow reduction, and ischemia/reperfusion injury. 
Excessive inflammatory responses may result in tissue injury. Finally, continuous inflammatory responses lead to multiple organ injury [3-5].

Recently, several investigators have linked the excessive generation of nitric oxide (NO) to lipopolysaccharide (LPS)-induced hypotension, vascular hyporeactivity, and death, suggesting that overproduction of NO plays an important role in septic shock [6,7]. LPS-dependent induction of inducible NO synthase (iNOS) is responsible for the overproduction of NO in circulatory shock [8]. This enzyme is expressed in many types of cells, such as neutrophils, macrophages, endothelial cells, vascular smooth muscle cells, mesangial cells, and chondrocytes [9]. The triggers for iNOS include LPS, interferon- $\gamma$, and many kinds of proinflammatory cytokines (e.g., TNF- $\alpha$ ) [10].

LPS activates macrophages causing the generation of free radicals, including the superoxide anion $\left(\mathrm{O}_{2}^{-}\right)$, hydrogen peroxide $\left(\mathrm{H}_{2} \mathrm{O}_{2}\right)$, and the hydroxyl radical $(\mathrm{OH})$, leading to oxidative damage in many tissues such as the liver [11]. NO can react with $\mathrm{O}_{2}^{-}$to form the peroxynitrite anion $\left(\mathrm{ONOO}^{-}\right)$, which results in severe oxidative damage leading to multiple organ failure and septic shock with high mortality $[12,13]$. Previous study showed that the inhibition of NOS activity by a selective iNOS inhibitor can attenuate the hepatic, renal, and pancreatic dysfunction associated with LPS-induced endotoxemia in rats [14]. Therefore, the overproduction of $\mathrm{NO}$ and NO-dependent free radicals seems to be related to development of multiple organ failure in endotoxic shock.

Dextromethorphan (DM), a dextrorotatory morphinan, is widely used clinically as a nonopioid anti-tussive [15]. However, the exact mechanism of its cough suppression action remains unclear. DM has been shown to be neuroprotective in several animal neurodegenerative models through inhibition of $N$-methyl-D-aspartate (NMDA) receptors $[16,17]$. Nevertheless, recent studies demonstrated that micromolar concentrations of DM reduce the inflammation-mediated degeneration of dopaminergic neurons through the inhibition of microglial activation [18]. The neuroprotective effect elicited by femtomolar concentrations of DM is mediated through the inhibition of LPS-induced proinflammatory factors, especially superoxide [19]. These studies suggest that the anti-inflammatory property of DMs may underlie its protective effect. LK4 (Figure 1), an analog of DM, has greater potency of anti-convulsant/neuron-protective actions than DM has [20]. In our preliminary study, we found that LK-4 can afford more protection against circulatory failure induced by LPS than that of DM. The efficacy of LK-4 in improvement of hemodynamic changes (hypotension and bradycardia) is about 2-fold higher than DM (data not shown). Thus, the aim of this study is to evaluate the anti-inflammatory effect of LK-4 in LPS-induced sepsis in vivo.

\section{Materials and methods}

\section{Animal preparation}

Male Wistar-Kyoto rats (250-300 g) were purchased from National Laboratory Animal Breeding and Research Center of National Science Council, Taiwan. All of animals were housed at an ambient temperature of $23 \pm 1{ }^{\circ} \mathrm{C}$ and moisture of $55 \pm 5 \%$. The rats were anesthetized by intraperitoneal injection of urethane $(1.2 \mathrm{~g} / \mathrm{kg})$. The trachea was cannulated to facilitate respira-

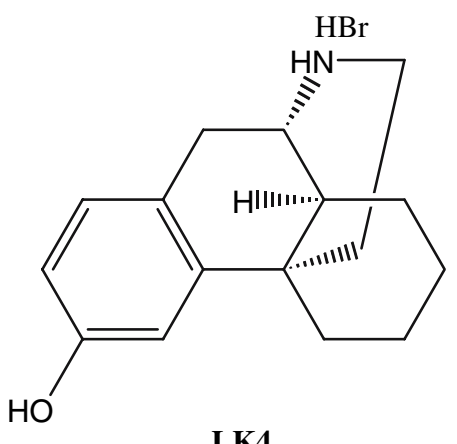

LK4

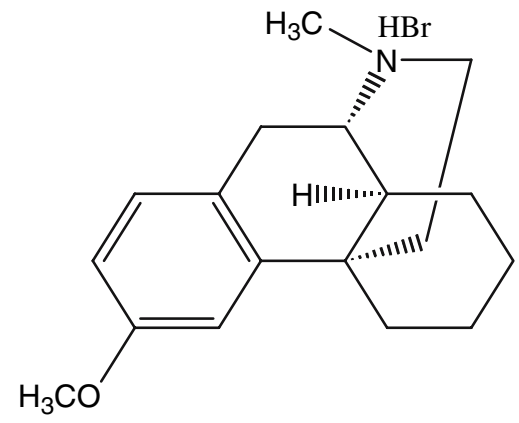

Dextromethorphan

Figure 1. The structure of LK-4 and dextromethorphan. 
tion. The left femoral artery was cannulated with polyethylene-50 (PE-50) and connected to a pressure transducer (P231D, Statham, Oxnard, CA, USA) for measurement of mean arterial pressure (MAP) and heart rate (HR), which were displayed on a Gould model TA5000 polygraph recorder (Gould, Valley View, OH, USA). The left femoral vein was cannulated for administration of drugs. After the surgical procedure was completed, all of cardiovascular parameters were allowed to stabilize for $30 \mathrm{~min}$.

\section{Drug administration}

Animals were randomly assigned into the following groups: (1) control group: rats were treated with vehicle (saline; i.v.); (2) LK-4 group: rats were treated with LK-4 (1-5 mg/kg, i.v.); (3) LPS group: rats were treated with $E$. coli lipopolysaccharide 3129 (LPS) $10 \mathrm{mg} / \mathrm{kg}$ (i.v.) [21]; (4) LK-4posttreatment group (LPS/LK-4): rats were treated with LK-4 (1-5 mg/kg, i.v.) at $30 \mathrm{~min}$ after LPS $(10 \mathrm{mg} / \mathrm{kg}$; i.v.) administration.

After recording the baseline hemodynamic parameters, animals were injected with vehicle or LPS and were monitored for $8 \mathrm{~h}$. Prior to (at time 0 ) and every hour after vehicle or LPS administration, $0.5 \mathrm{ml}$ of blood was withdrawn to measure the level of TNF- $\alpha$ and nitrate. Any blood withdrawn was immediately replaced by an injection of an equal volume of saline (i.v.) in order to maintain the blood volume. Blood samples were centrifuged for $5 \mathrm{~min}$ at $12,000 \times \mathrm{g}$. Plasma samples were stored at $-80{ }^{\circ} \mathrm{C}$ until analysis. At the end of the $8 \mathrm{~h}$ experiment, thoracic aortas of each group were dissected out for vascular responses or superoxide analysis.

\section{Organ bath experiments}

The thoracic aortas were cleared of adhering periadventitial fat and cut 3-4 $\mathrm{mm}$ in length. The rings were mounted in $20 \mathrm{ml}$ organ baths filled with $37{ }^{\circ} \mathrm{C}, 95 \% \mathrm{O}_{2} / 5 \% \mathrm{CO}_{2}$ oxygenated Kreb's solution ( $\mathrm{pH}$ 7.4) consisting of $(\mathrm{mM})$ : $\mathrm{NaCl} 118$, $\mathrm{KCl}$ 4.7, $\mathrm{NaHCO}_{3} 25, \mathrm{KH}_{2} \mathrm{PO}_{4} 1.2, \mathrm{MgSO}_{4} 1.2$, $\mathrm{CaCl}_{2} 2.5$, glucose 11. Isometric force was measured with Grass FT03 type transducers (Grass instruments, Quincy, MA, USA) and recorded on a MacLab Recording and Analysis System (AD Instrument, Castle Hill, Australia). In the rings,
$2 \mathrm{~g}$ passive tension was applied and equilibrated for 60-90 min. After rings were stable under setting, the ring was added $0.1 \mu \mathrm{M}$ norepinephrine (NE) to contract maximally, then acetylcholine (ACh) $1 \mu \mathrm{M}$ was added to relax. If (contractile tension-relaxation tension)/contractile tension is $>80 \%$, it reveals that the endothelium is intact. Kreb's solution in organ baths was changed every 15 min for 2-3 times. After the rings with (contractile tension-relaxation tension)/contractile tension $>80 \%$ were stable toward baseline tension level then the next steps were performed. (1) Concentration-response curves of NE: different concentrations of NE $(10 \mathrm{nM}-10 \mu \mathrm{M})$ were added into the organ bath and the tension change was monitored by force-displacement transducer, and recorded by computer. Kreb's solution was used to wash two times every 15 min for the next step; (2) Concentration-response curves of ACh: NE $(1 \mu \mathrm{M})$ for maximum contraction, then $\mathrm{ACh}$ was added accumulatively from $10 \mathrm{nM}$ to $10 \mu \mathrm{M}$ for relaxation recording. Then Kreb's solution was changed two times every 15 min for the next step; (3) Concentration-response curves of L-arginine: $\mathrm{NE}(1 \mu \mathrm{M})$ for maximum contraction, then L-arginine $(10 \mathrm{nM}-10 \mu \mathrm{M})$ was added accumulatively for relaxation recording.

\section{Quantification of organ function and injury}

At 0,6 , and $8 \mathrm{~h}$ after the injection of LPS, blood samples were collected. The blood samples were centrifuged $12,000 \times g$ to prepare serum. All serum was analyzed within $24 \mathrm{~h}$. Eighty microliters of plasma was used to analyze liver or kidney functions. The following marker enzymes measured in the plasma were regarded as biochemical 6060 indicators of multiple organ dysfunction or failure. Liver dysfunction and failure were assessed by measuring the rises in plasma levels of GPT (a specific marker for hepatic parenchymal injury), GOT (a nonspecific marker for hepatic parenchymal injury). Renal dysfunction and failure were assessed by measuring the rises in plasma levels of creatinine (an indicator of reduced glomerular filtration rate and, hence, renal failure), and BUN (an indicator of impaired excretory function of the kidney and/or increased catabolism). All of these biochemical parameters were analyzed within 15 min by Fuji DRI-CHEM 3030 (Fuji Photo Film Co., Ltd., Tokyo, Japan). 


\section{Histopathological studies}

The vital organs, including the lung, liver, and kidney, were harvested at $8 \mathrm{~h}$ for histopathological studies, as previously described [21, 22]. These organ tissues were fixed in buffered formaldehyde $(10 \%$ in phosphate-buffered saline) for more than $8 \mathrm{~h}$. The fixed organs were dehydrated in graded ethanol and embedded in paraffin (Tissue-processor, Japan). Four-micrometer sections were cut (sliding microtome, Leica Jung SM 2000) and removed paraffin by xylene. Then, the tissue sections were stained with the hematoxylin and eosin reagent for light microscopy. This histologic alteration was quantitatively analyzed as an index of the severity of tissue injury. The index was determined by counting the numbers of polymorphonuclear neutrophil (PMN) in 10 randomly selected high-power fields and by the histologic changes (e.g., interstitial edema and/or congestion) evaluated by a pathologist in a blinded fashion.

\section{Measurement of plasma TNF- $\alpha$ concentration}

The blood sample $(0.5 \mathrm{ml})$ was collected at $0,1,2$, and $4 \mathrm{~h}$ after the injection of LPS for measurement of the TNF- $\alpha$ level in plasma by an enzyme-linked immunoadsorbent assay (mouse TNF- $\alpha$ ELISA Kit, Genzyme Co., Cambridge, MA, USA), as previously described [21].

\section{Plasma nitrite/nitrate determination}

A sample of $30 \mu \mathrm{l}$ thawed plasma was deproteinated with $100 \mu \mathrm{l} 95 \%$ alcohol for $30 \mathrm{~min}$ $\left(4{ }^{\circ} \mathrm{C}\right)$. Subsequently, these serum samples were centrifuged for $6 \mathrm{~min}$ at $12,000 \times g$. The supernatant $(6 \mu \mathrm{l})$ was injected into a collection chamber containing $5 \% \mathrm{VCL}_{3}$. In this strong reducing environment, both nitrate and nitrite were converted to NO. A constant stream of helium gas carried NO into a NO analyzer (Sievers 280NOA; Sievers Instruments Inc., Boulder, CO, USA), where the NO reacted with ozone $\left(\mathrm{O}_{3}\right)$, resulting in the emission of light. Light emission is proportional to the NO formed. Standard amounts of sodium nitrate were used for calibration (Sigma Chemical Co., St. Louis, MO, USA).

\section{Western blot analysis of iNOS protein expression in lungs}

At $8 \mathrm{~h}$ after the injection of saline or LPS, the experimental animals were sacrificed. The lung tissues were obtained from rats in control, LK-4, LPS, and LK-4-posttreatment groups, and frozen at $-80{ }^{\circ} \mathrm{C}$ before assay. Frozen samples were ground in a mortar containing liquid nitrogen. The powdered tissue was suspended in $1 \mathrm{ml}$ of lysis buffer $(50 \mathrm{mM}$ HEPES, $5 \mathrm{mM}$ EDTA, and $50 \mathrm{mM} \mathrm{NaCl}, \mathrm{pH}$ 7.5) containing protease inhibitors $(10 \mu \mathrm{g} / \mathrm{ml}$ of aprotinin, $1 \mathrm{mmol} / 1 \mathrm{PMSF}$, and $10 \mu \mathrm{g} / \mathrm{ml}$ of leupeptin) and agitated at $4{ }^{\circ} \mathrm{C}$ for $1 \mathrm{~h}$. After centrifugation at $10,000 \times g$ at $4{ }^{\circ} \mathrm{C}$ for $30 \mathrm{~min}$, the protein concentration in the supernatant was determined using BCA protein assay kit (Pierce, Rockford, IL, USA).

Samples containing equal amounts of protein were loaded onto $10 \%$ sodium dodecyl sulfatepolyacrylamide gels and subjected to electrophoresis, and subsequently blotted onto nitrocellulose membrane (Millipore, Bedford, USA). Membranes were blocked with Tris-buffered saline buffer (TBS), pH 7.4, containing $0.1 \%$ Tween-20 and $5 \%$ skim milk, then incubated overnight at $4{ }^{\circ} \mathrm{C}$ with various primary antibodies in TBS containing $0.1 \%$ Tween-20. The antibodies included mouse anti-iNOS (1:1000 dilution, Stressgen Biotechnologies Co., Victoria, BC, Canada), mouse anti- $\beta$-actin (1:2000 dilution, Sigma-Aldrich, St. Louis, MO, USA). The membranes were incubated with the horseradish peroxidase (HRP)-conjugated secondary antibodies (1:1000 dilution, Cell Signaling). The blots were detected with an enhanced chemiluminescence kit (Pierce, Rockford, IL, USA), and the membrane exposed to X-ray film (Kodak, Rochester, NY, USA) for $5 \mathrm{~min}$. The density of the respective bands was quantified by densitometric scanning of the blots using Image-Pro software (Media Cybemetrics, Inc).

\section{Aortic superoxide anion detection by chemiluminescence}

Detection of superoxide anions was performed as described previously [23]. Thoracic aorta was cut into $3-4 \mathrm{~mm}$ rings and incubated in $95 \% \mathrm{O}_{2} / 5 \%$ $\mathrm{CO}_{2}$ oxygenated modified Kreb's/HEPES solution $\left(37^{\circ} \mathrm{C}\right)$ for $30 \mathrm{~min}$. Then the aorta sections were 
put into a 96-well plate in which every well was filled with $200 \mu$ l modified Kreb's/HEPES solution, and placed in a luminescence measurement system (Microplate Luminometer LB 96 V, Berthold, Germany). It can perform auto-injection of $250 \mu \mathrm{M}$ lucigenin (final volume of $250 \mu \mathrm{l}$ ) into the vessels for interacting with superoxide. Counts were obtained at $15 \mathrm{~min}$ intervals at room temperature. After recording was complete, the vessel ring was dried in a $95{ }^{\circ} \mathrm{C}$ oven for $24 \mathrm{~h}$. The results were expressed as relative units of luminescence (RUL) per 15 min per milligram dry weight vessel (i.e., RUL/15 $\mathrm{min} / \mathrm{mg}$ ).

\section{Survival rate study}

The ICR mice $(25-30 \mathrm{~g})$ were divided into four groups, LPS was administrated intraperitoneally at a dose of $70 \mathrm{mg} / \mathrm{kg}$. LK-4 (5 mg/kg, i.p.) was administrated intraperitoneally at $30 \mathrm{~min}, 6$ and $12 \mathrm{~h}$ after LPS administration. Survival of mice was monitored for $36 \mathrm{~h}$.
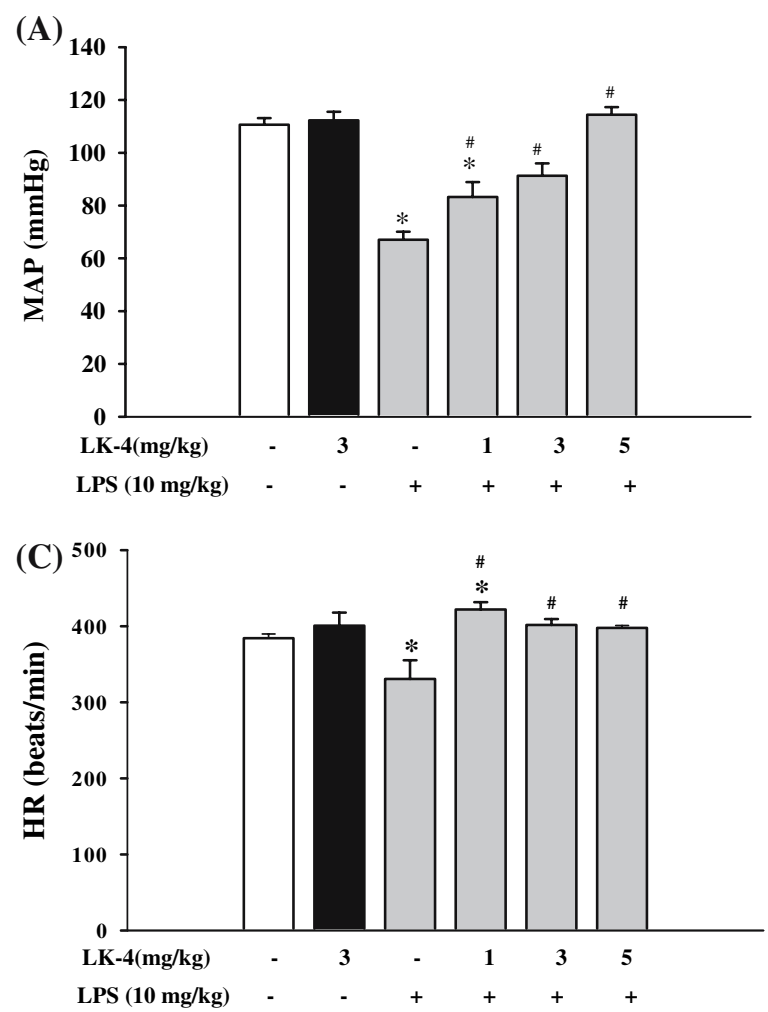

\section{Statistical analysis}

Data are expressed as mean \pm SE. One-way ANOVA was performed in the statistical analysis of data. When group comparisons showed a significant difference, the Student-Newman Keuls test was used. A value of $p<0.05$ was accepted to indicate statistical significance. Comparisons among mortality rates of the groups were made with the Fisher's exact test.

\section{Results}

\section{Effects of $L K-4$ on MAP and $H R$ in rats with endotoxemia}

In order to determine a suitable dose of LK-4 for use in this study, a dose-response study was carried out, results as shown in Figure 2A and B. In the control group, mean values for mean arterial pressure (MAP) and heart rate (HR) were
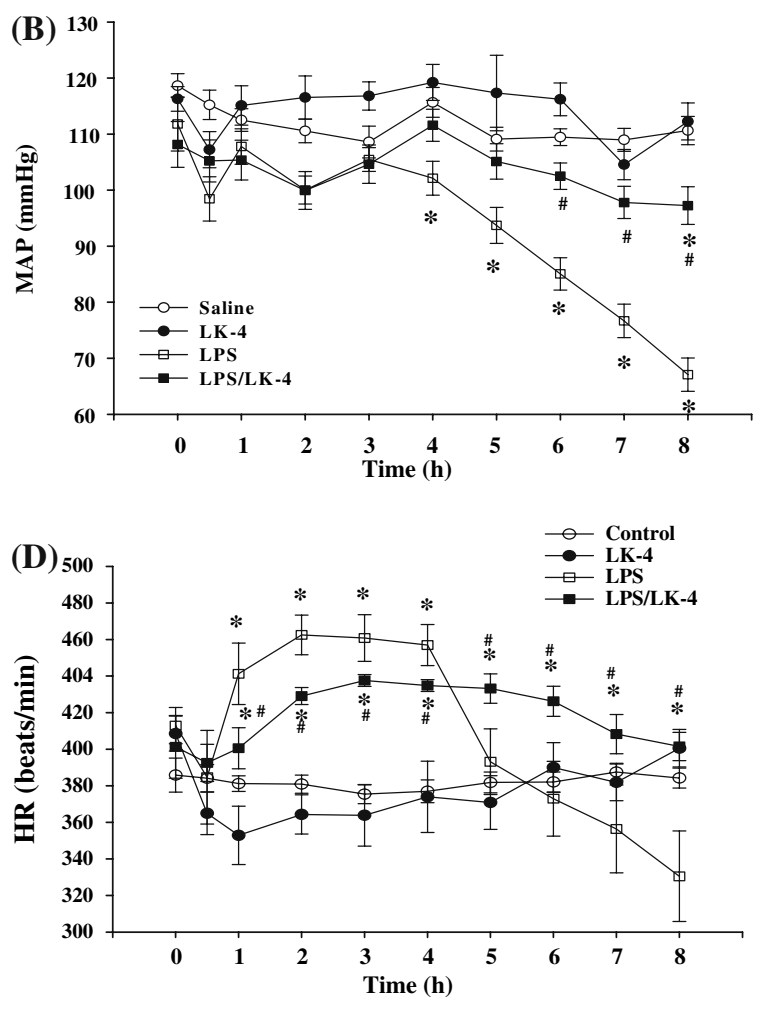

Figure 2. Effects of posttreatment with LK-4 (1-5 mg/kg, i.v.) on the MAP (A) and HR (B) in rats treated with LPS (10 mg/kg, i.v.) for $8 \mathrm{~h}$. The effect of LK-4 (3 mg/kg, i.v.) on time course of MAP (C) and HR (D) changes after LPS administration for $8 \mathrm{~h}$. Data represent as mean $\pm \operatorname{SE}(n=6) .{ }^{*} p<0.05$ : LPS vs. control, $\# p<0.05$ : LPS/LK-4 vs. LPS. 

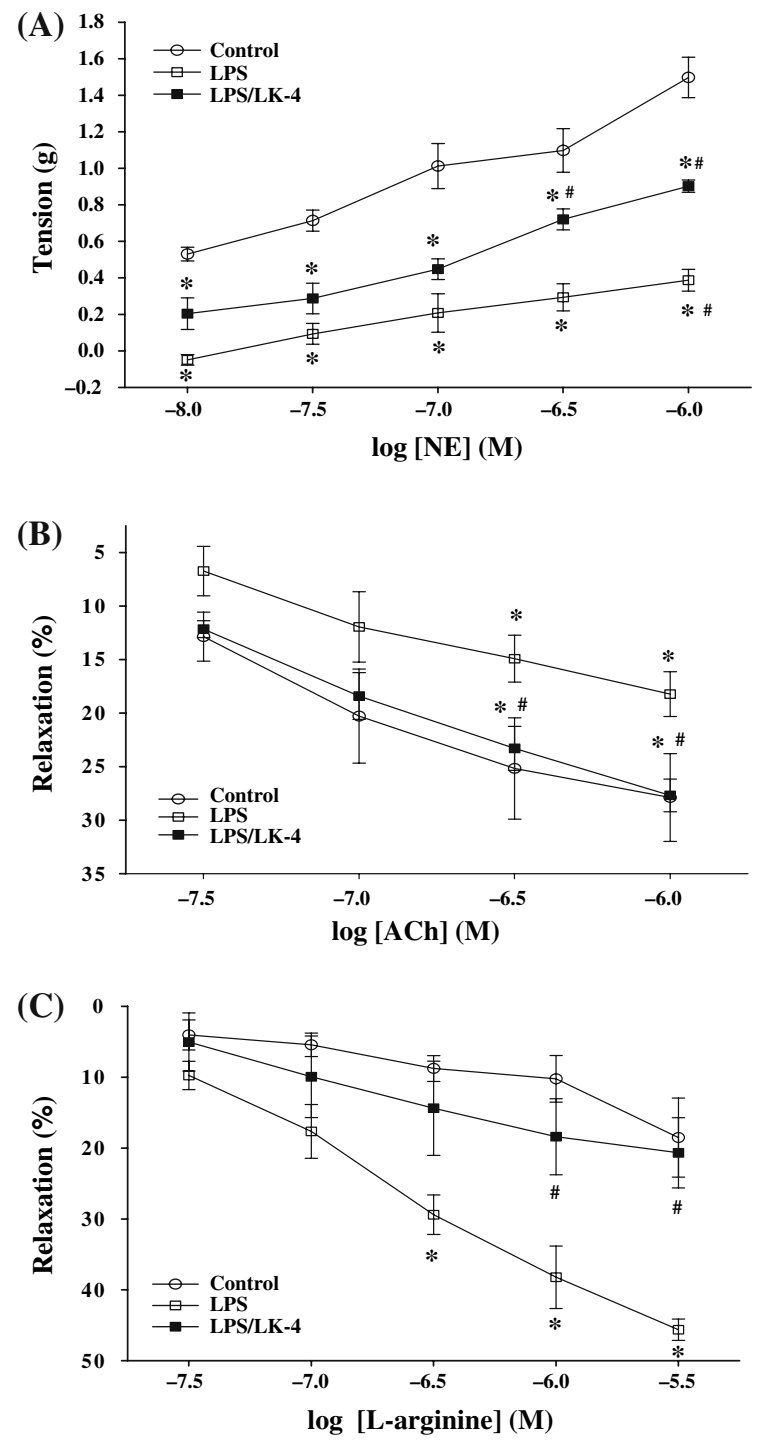

Figure 3. Effects of posttreatment with LK-4 $(3 \mathrm{mg} / \mathrm{kg})$ on the concentration-response curves of NE (A), ACh (B), and L-arginine $(\mathrm{C})$ in aortic rings from LPS-treated rats. Data represent mean $\pm \mathrm{SE} \quad(n=6) . * p<0.05$ : LPS vs. control, $\# p<0.05:$ LPS/LK-4 vs. LPS.

$111 \pm 3 \mathrm{mmHg}$ and $384 \pm 5$ beats $/ \mathrm{min}$, respectively. After $8 \mathrm{~h}$ of LPS $(10 \mathrm{mg} / \mathrm{kg})$ administration, MAP and HR reduced to $67 \pm 3 \mathrm{mmHg}$ and $331 \pm 25$ beats/min, respectively. Treating with LK-4 (3 or $5 \mathrm{mg} / \mathrm{kg}$ ) at $30 \mathrm{~min}$ after the administration of LPS, MAP, and HR maintained significantly higher levels at $8 \mathrm{~h}$ than the LPStreated group. In addition, LK-4 (3 mg/ $/ \mathrm{kg})$ alone had no significantly effects on either MAP or HR. Thus, this dose of $3 \mathrm{mg} / \mathrm{kg}$ of LK-4 was chosen for the following studies.
Figure 2C and D illustrated the time course of changes in MAP and HR. The baseline MAP of four groups were about $108 \pm 6-118 \pm 3 \mathrm{mmHg}$ and did not show significant differences among groups. The administration of LPS resulted in a rapid decrease in MAP from $112 \pm 4-98 \pm 4 \mathrm{mmHg}$ within $30 \mathrm{~min}$. The MAP then slowly returned to $108 \pm 3 \mathrm{mmHg}$ at $1 \mathrm{~h}$, and gradually decreased to $67 \pm 3 \mathrm{mmHg}$ at the end of the experiment $(8 \mathrm{~h})$. The MAP of LK-4-posttreatment group at 6-8 h after LPS administration was significantly greater than that of the LPS group $(p<0.05)$ (Figure 2C).

The mean baseline values of HR in four groups were about $386 \pm 9-413 \pm 10$ beats/min and there was no significant difference among groups. In the LPS group, HR progressively increased and peaked at $2-4 \mathrm{~h}$, and then decreased lasting to the end of the experiment. However, posttreatment with LK-4 $(3 \mathrm{mg} / \mathrm{kg})$ prevent LPS-induced tachycardia during $1-4 \mathrm{~h}$, and prevented LPS-induced bradycardia at 5-8 h (Figure 2D).

\section{Effects of LK-4 on NE-induced vasoconstriction,} $A C h$ - and L-arginine-induced vasorelaxation, in vitro

Results in Figure 3 showed that vascular hyporeactivity of NE and ACh were found in the LPS group. This hyporesponse to $\mathrm{NE}$ and $\mathrm{ACh}$ were significantly reversed by posttreatment with LK-4 $(3 \mathrm{mg} / \mathrm{kg}$, i.v. $)(p<0.05$, Figure $3 \mathrm{~A}$ and B). However, LPS significantly enhanced L-arginine-induced vasorelaxation, and LK-4 posttreatment significantly reversed the effect of LPS (Figure 3C).

Effect of LK-4 on liver function (GOT and GPT)

Baseline values of GPT and GOT were not significantly different among groups (Figure 4A and B). LPS caused a significant increase in the plasma levels of GPT and GOT at the late stage (6 and $8 \mathrm{~h}$ ). The rise in plasma levels of GPT and GOT caused by LPS were reduced by the posttreatment of rats with LK-4.

\section{Effect of LK-4 on renal function (creatinine and $B U N)$}

Baseline values of creatinine and BUN were not significantly different among groups (Figure 4C and D). LPS caused a significant increase of 

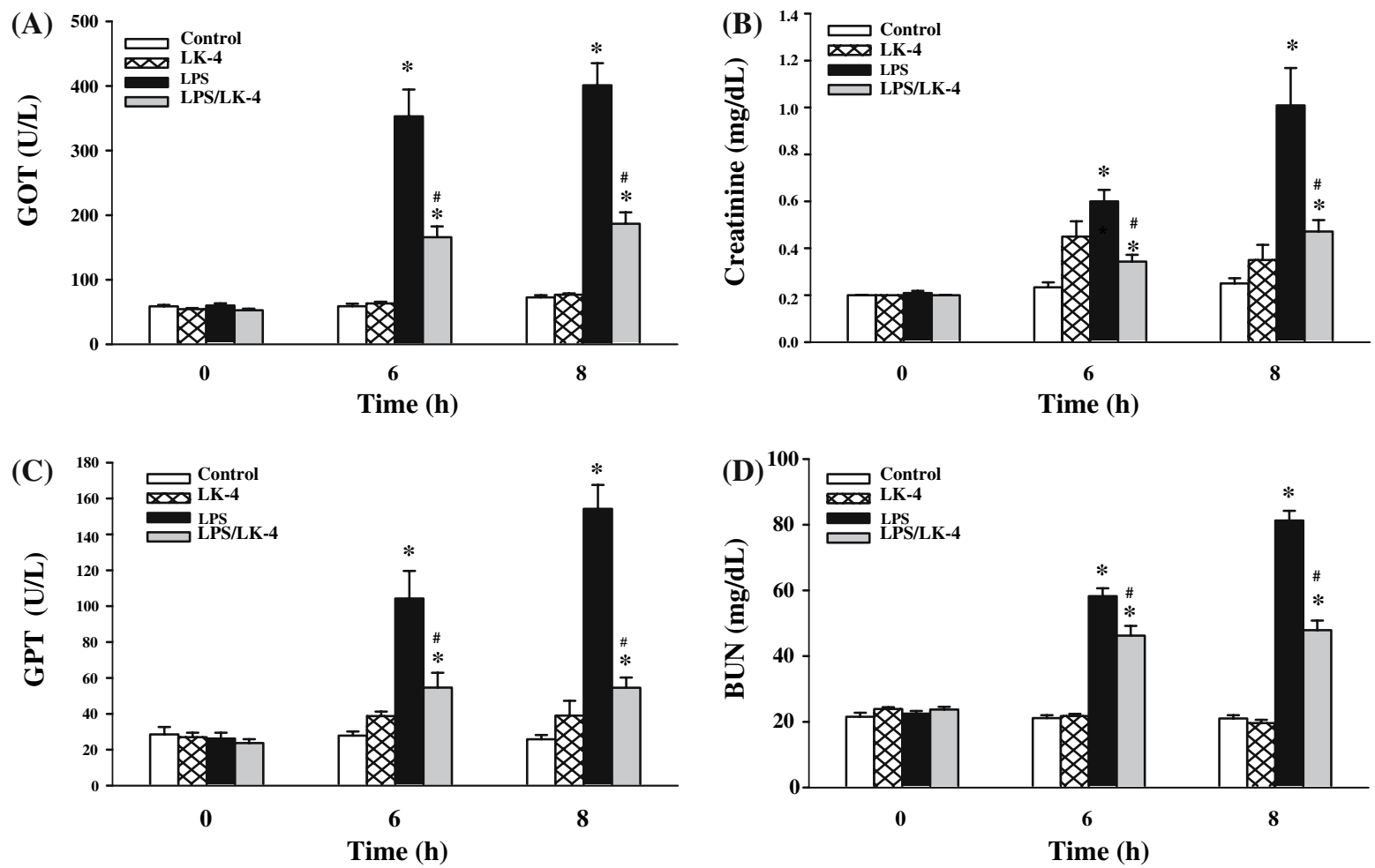

Figure 4. Effects of LK-4 (3 mg/kg) posttreatment on plasma levels of GOT (A), GPT (B), creatinine (C), and BUN (D) in rats treated with LPS. Data represent mean $\pm \mathrm{SE}(n=6) .{ }^{*} p<0.05$ : LPS vs. control, $\# p<0.05$ : LPS/LK-4 vs. LPS.

creatinine and BUN level at the late stage $(6$ and $8 \mathrm{~h}$ ). The rise in plasma levels of creatinine and BUN caused by LPS were reduced by the posttreatment of rats with LK-4.

\section{Effect of LK-4 on plasma TNF- $\alpha$ level}

The basal plasma levels of TNF- $\alpha$ were not significantly different among groups. LPS caused a significant increase in plasma TNF- $\alpha$ level, which reached a peak at $1-2 \mathrm{~h}$ after LPS injection and subsequently decreased slowly. LK-4 alone did not cause an increase in the plasma TNF- $\alpha$ level. However, posttreatment with LK-4 (3 mg/ $\mathrm{kg})$ significantly decreased the LPS-induced increase of plasma TNF- $\alpha$ level at $1-2 \mathrm{~h}$ as compared with the LPS group (Figure 5A).

\section{Effect of LK-4 on superoxide anion formation}

The content of superoxide anions in thoracic aorta after $8 \mathrm{~h}$ LPS treatment was significantly higher than that of the control group $(2480 \pm 373$ vs. $337 \pm 19.6$ RLU, $p<0.05)$. However, posttreat- ment with LK-4 (3 mg/kg) significantly reduced superoxide anion formation as compared with the LPS group $(720 \pm 142.5$ vs. $2480 \pm 373$ RLU, $p<0.05$ ) (Figure 5B).

\section{Effect of LK-4 on plasma nitrite/nitrate content}

In the control and LK-4 alone groups, the level of plasma nitrite/nitrate did not significantly change through out the experimental period. LPS treatment significantly induced the elevation of plasma nitrite/nitrate content at $4-8 \mathrm{~h}$ as compared with the control group $(p<0.05)$. However, posttreatment of LK-4 $(3 \mathrm{mg} / \mathrm{kg})$ significantly suppressed this LPS-induced increase in plasma nitrite/nitrate levels $(p<0.05)$ (Figure 5C).

\section{PMN infiltration}

In the control group, light microscopy showed no infiltration of PMNs in the lungs or liver. In contrast, increased infiltration of PMNs in both lungs and liver was noted at $8 \mathrm{~h}$ after injection of LPS. In rats posttreated with LK-4, infiltration of 

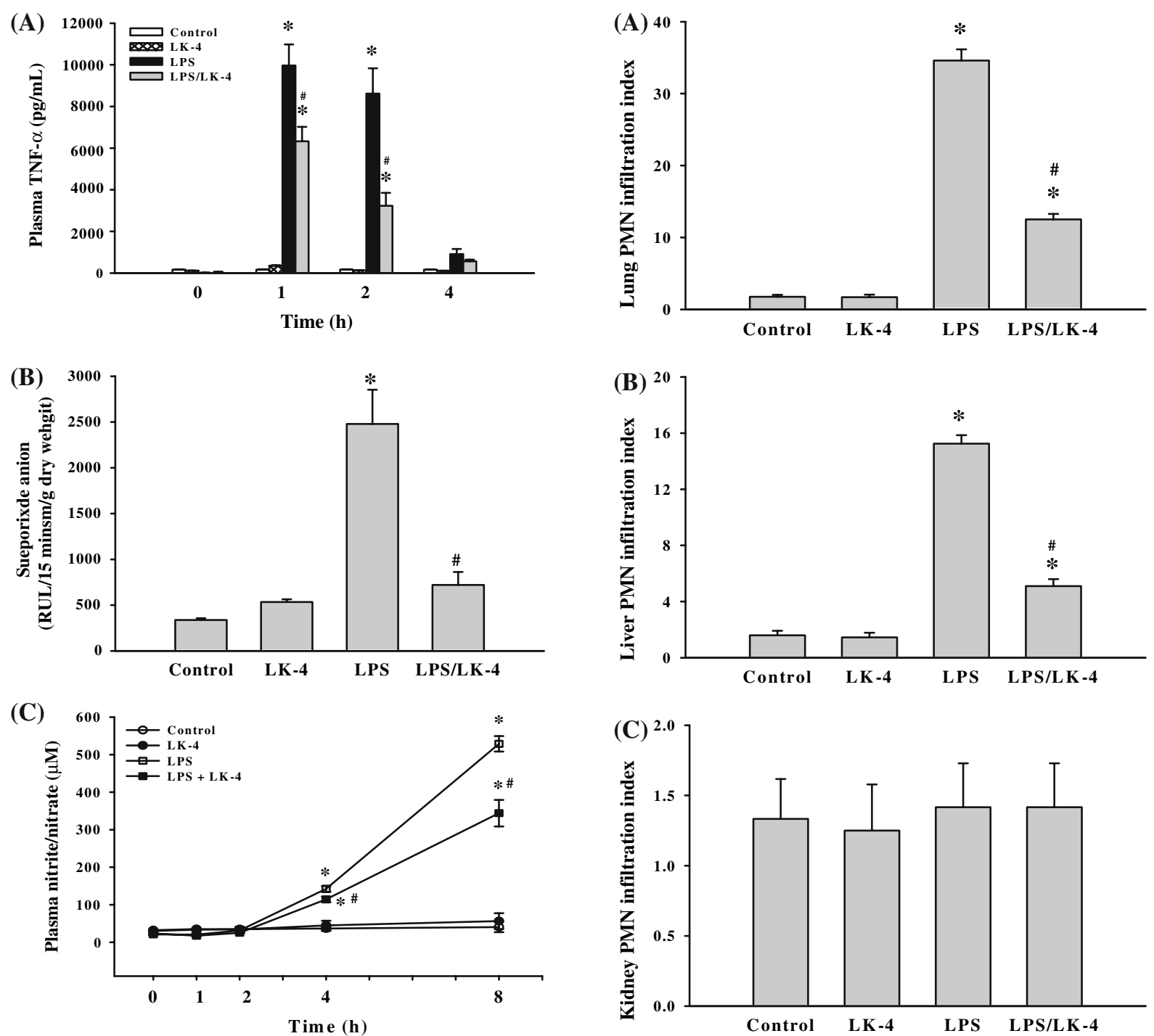

Figure 5. Effects of posttreatment with LK-4 $(3 \mathrm{mg} / \mathrm{kg})$ on plasma TNF- $\alpha$ level (A), superoxide anion formation in aortic tissues (B), and plasma nitrite/nitrate $(\mathrm{C})$ from rats treated with LPS for $8 \mathrm{~h}$. Data represent as mean $\pm \operatorname{SE}(n=6)$. ${ }^{*} p<0.05$ : LPS vs. control, $\# p<0.05$ :LPS/LK-4 vs. LPS.

PMNs in lung and liver was significantly reduced (Figure 6A and B). However, there was no significant difference in infiltration of PMNs in kidneys among groups (Figure 6C).

Effect of LK-4 on expression of iNOS protein in lungs

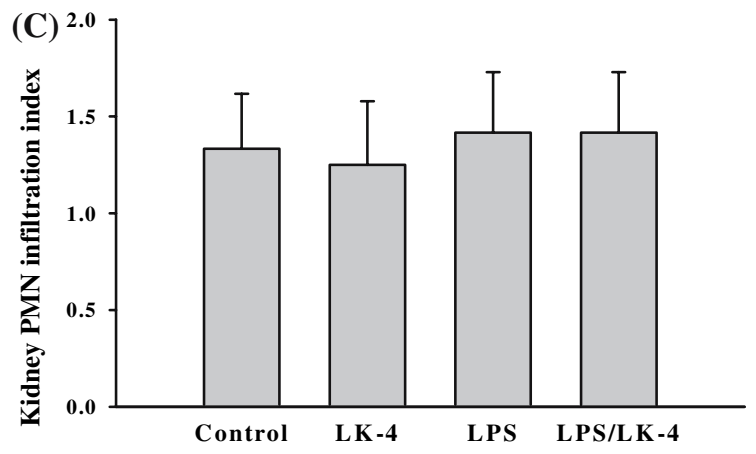

Figure 6. Effects of LK-4 $(3 \mathrm{mg} / \mathrm{kg})$ posttreatment on the PMN infiltration index in the lung (A), liver (B), and kidney (C) of rats treated with LPS. Data represent as mean $\pm \mathrm{SE}$ $(n=6) .{ }^{*} p<0.05$ : LPS vs. control, $\# p<0.05$ :LPS/LK-4 vs. LPS.

iNOS protein was observed in rats treated with LPS for $8 \mathrm{~h}$. Posttreatment of rats with LK-4 $(3 \mathrm{mg} / \mathrm{kg})$ significantly reduced the induction of iNOS challenged with LPS.

Effect of LK-4 on survival rate of mice treated with LPS

As shown in Figure 8, the survival rate reduced to $12.5 \%$ after the administration of a high dose of LPS (70 mg/ $\mathrm{kg}$ ) to ICR mice for $36 \mathrm{~h}$. However, in 

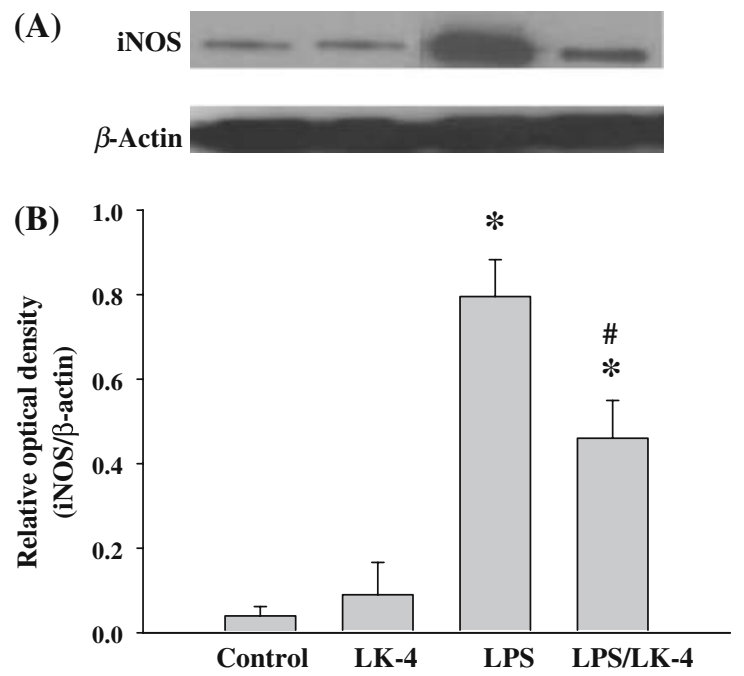

Figure 7. Effects of posttreatment with LK-4 $(3 \mathrm{mg} / \mathrm{kg})$ on iNOS protein expression in the lung from rats treated with LPS for $8 \mathrm{~h}$. Depicted is a typical display of iNOS protein expression (upper panel) and the statistical analysis of the changes of iNOS protein (lower panel). Data represent as mean $\pm \operatorname{SE}(n=6) .{ }^{*} p<0.05$ : LPS vs. control, $\# p<0.05$ : LPS/LK-4 vs. LPS.

the LK-4-posttreatment group, the survival rate of mice increased to $43.75 \%$ when compared with the LPS group $(p<0.05)$.

\section{Discussion}

In the present study, we demonstrate that posttreatment with LK-4, an analog of dextro-

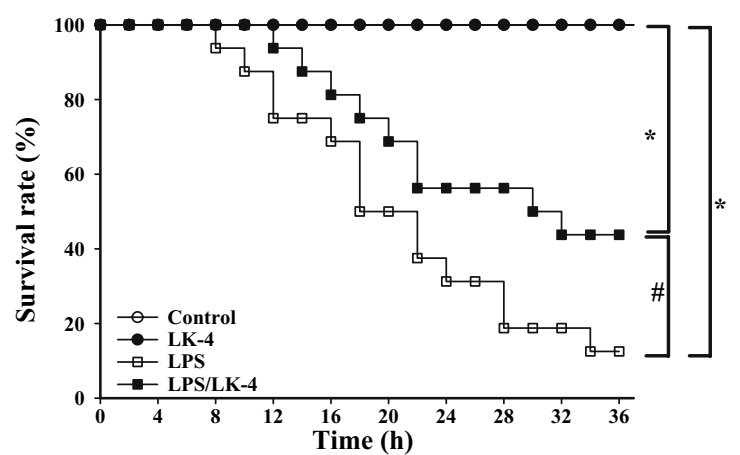

Figure 8. Effects of LK-4 on the survival rate of LPS-treated mice. Each group consisted of 12 animals. Vehicle (saline) was injected in control group. LPS $(70 \mathrm{mg} / \mathrm{kg})$ was injected (i.p.) in LPS-treated group. LK-4 (5 mg/kg, i.p.) was injected at $30 \mathrm{~min}, 6$ and $12 \mathrm{~h}$ after LPS injection in LPS/LK-4 group. $* p<0.05$ : LPS or LPS/LK-4 vs. control, $\# p<0.05$ : LPS/LK4 vs. LPS. methorphan, possesses the therapeutic effect on LPS-induced septic shock in rats. LK-4 improved circulatory function evidenced by preventing hypotension as well as bradycardia, and preserving vascular contracture ability as well as endothelial function in late stage. It also improved hepatic and renal function during sepsis, accompanied with attenuation of pulmonary PMN infiltration. Furthermore, LK-4 increased the survival rate of mice insulted by toxic dose of LPS. These beneficial effects may be associated with the anti-oxidant and anti-inflammatory actions of LK-4 by suppression of free radical formation, $\mathrm{TNF}-\alpha$ production, and iNOS induction.

Sepsis is a systemic response to infection, and septic shock is one of the most common causes of death in intensive care units [24]. The most common cause of sepsis is an exposure to the structural component of a Gram-negative bacterial membrane LPS and key symptoms include hypotension and vasoplegia, which may lead to multiple organ dysfunction and ultimately death [25]. Bacterial LPS in the bloodstream induces the overexpression of various inflammatory mediators, such as interleukin- $1 \beta$, TNF- $\alpha$, NO, and prostaglandin E2 (PGE2). A large amount of inflammatory mediators produced in the body are thought to contribute to the LPS-induced symptoms of septic shock and mortality [26]. Among these inflammatory mediators; NO is known to be closely associated with hypotension and hyporesponsiveness to vasoconstrictor stimuli in endotoxin-induced sepsis $[24,25]$. In the present study, LK-4 increased NE-induced vasocontraction, and ACh-induced vasorelaxation (Figure $3 \mathrm{~A}$ and $\mathrm{B}$ ), indicating that it improves vascular contracture ability and preserve endothelium function in sepsis. Moreover, LK-4 reduced the L-arginineinduced relaxation after LPS treatment (Figure 3C), implying that it could suppress the activity of iNOS. This point was further supported by the results in which LK-4 reduced plasma nitrate/nitrite concentration and iNOS protein expression in lung tissue challenged by LPS (Figures 5C and 7).

Production of NO by iNOS is beneficial in fighting bacteria, but its overproduction can be harmful as shown in endotoxic shock [27]. There is increasing evidence that overproduction of TNF- $\alpha$ during infection also leads to severe systemic toxicity and even death [28]. Evidence supporting 
this hypothesis comes from reports indicating that mediators (e.g. TNF- $\alpha$ and interleukins) produced by endotoxin challenge can induce iNOS expression and produce large amounts of NO [29, 30]. In the present study, the elevation of plasma TNF- $\alpha$ level by LPS was downregulated by LK-4 treatment (Figure 5A and $\mathrm{C}$ ), indicating that this antiinflammatory activity of LK-4 may contribute to the increased survival rate of mice and improve hyporeactivity and circulatory function in endotoxemic rats.

In sepsis, abundant reactive oxygen species are produced, and several sources of oxygen radical species have been proposed as being the cause of tissue damage. Following transmigration and activation, infiltrating neutrophils produce abundant oxygen radicals via oxidative bursts. Other sources of oxygen radical species include activated macrophages and various extracellular molecular processes such as arachidonic acid metabolism and xanthine dehydrogenase oxidation [31]. NO may combine with $\mathrm{O}_{2}^{-}$to form the more-potent reactive oxygen metabolite, the peroxynitrite anion $\left(\mathrm{ONOO}^{-}\right)$, which decomposes to form $\mathrm{OH}$ [12]. Both $\mathrm{ONOO}^{-}$and $\mathrm{OH}$ are responsible for cellular lipid peroxidation, protein oxidation, and mitochondrial impairment function, which cause further damage to tissues and can induce cell death [32]. Results in this study demonstrated that posttreatment with LK-4 significantly suppressed the superoxide anion production in blood vessels induced by LPS (Figure 5B). Moreover, LPS induced multiple organ injuries/dysfunctions, which were further evidenced by histologic findings of PMN infiltration in the lung and liver. Treatment with LK-4 not only ameliorated the deterioration of hymodynamic changes (hypotension and bradycardia) but also attenuated liver and lung abnormalities (Figure 6A and B) caused by LPS. These results indicate that some of the beneficial effects of LK-4 may be associated with its antioxidant properties (as shown in Figure 5B). In addition, Gram-negative-related sepsis is frequently associated with acute renal failure, which is associated with the overproduction of ROS [33]. In the present study, results as shown in Figure 4C and D, also found LPS-induced kidney dysfunction, but the PMN infiltration in kidney was normal (Figure 6C). This discrepancy may result from kidney is more resistance to LPS, which needs to be further elucidated. The cellular mechanisms of action responsible for the inhibitory effects of LK-4 on TNF- $\alpha$ release, NO synthesis, and superoxide anion generation need to be further investigated.

In conclusion, LK-4 is capable of reducing circulatory failure and improves survival in animals with endotoxic shock. This therapeutic effect of LK-4 is associated with its anti-inflammatory effects by reduction in proinflammatory cytokine release, suppression of NO production, as well as its anti-oxidant properties. These finding may provide insights into the potential novel effect of LK-4 as a therapeutic agent against septic shock in the future. As for the detail of anti-inflammatory molecular mechanism of LK-4 on sepsis will further designed experiments to approach.

\section{Acknowledgement}

This work was supported in part by research grants from the National Science Council (NSC94-2320-B-016-002, Mao-Hsiung Yen), Taipei, Taiwan.

\section{Reference}

1. Bone R.C., Grodzin C.J. and Balk R.A., Sepsis, a new hypothesis for pathogenesis of the disease process. Chest 112: 235-243, 1997.

2. Angus D.C., Linde-Zwirbe W.T., Lidicker J., Clermont G., Carcillo J. and Pinsky M.R., Epidemiology of severe sepsis in the United States: analysis of incidence, outcome, and associated costs of care. Crit. Care Med. 29: 1303-1310, 2001.

3. Marik P.E. and Zalopa G.P., Therapeutic selection: has its time come?. Crit. Care Med. 30: 949-952, 2002.

4. Brix-Christensen V., Andersen S.K., Andersen R., Mengel A., Dyhr T., Andersen N.T., Larsson A., Schmitz O., Orskov H. and Tonnesen E., Acute hyperinsulinemia restrains endotoxin-induced systemic inflammatory response. Anesthesiology 100: 861-870, 2004.

5. Marix P.E. and Raghavan M., Stress-hyperglycemia, insulin and immunomodulation in sepsis. Intensive Care Med. 30: 748-756, 2004.

6. Moncada S., Palmer R.M. and Higgs E.A., Nitric oxide: physiology, pathophysiology, and pharmacology. Pharmacol. Rev. 43: 109-142, 1991.

7. Yang F., Comtois A.S., Fang L., Hartman N.G. and Blaise G., Nitric oxide-derived nitrate anion contributes to endotoxic shock and multiple organ injury/dysfunction. Crit. Care Med. 30: 650-657, 2002.

8. Szabo C., Salzman A.L. and Ischiropoulos H., Endotoxin triggers the expression of an inducible isoform of nitric oxide synthase and the formation of peroxynitrite in the rat aorta in vivo. FEBS Lett. 363: 235-238, 1995. 
9. Kirkebone K.A. and Stramd O.A., The role of nitric oxide in sepsis: an overview. Acta Anaesthesiol. Scand. 43: 275-288, 1999.

10. Jiang H., Stewart C.A. and Leu R.W., Tumor-derived factor synergizes with IFN-gamma and LPS, IL-2 or TNFalpha to promote macrophage synthesis of TNF-alpha and TNF receptors for autocrine induction of nitric oxide synthase and enhanced nitric oxide-mediated tumor cytotoxicity. Immunobiology 192: 321-342, 1995.

11. Sugino K., Dohi K., Yamada K. and Kawasaki T., Changes in the levels of endogenous antioxidants in the liver of mice with experimental endotoxemia and the protective effects of the antioxidants. Surgery 105: 200206, 1989.

12. Beckman J.S., Beckman T.W., Chert J., Marshall P.A. and Freeman B.A., Apparent hydroxyl radical production by peroxynitrite: implications for endothelial injury from nitric oxide and superoxide. Proc. Natl. Acad. Sci. USA 87: 1620-1624, 1990.

13. Deitch E.A., Multiple organ failure. Pathophysiology and potential future therapy. Ann. Surg. 216: 117-134, 1992.

14. Wu C.C., Ruetten H. and Thiemermann C., Comparison of the effects of aminoguanidine and $N$ omega-nitro-L-arginine methyl ester on the multiple organ dysfunction caused by endotoxaemia in the rat. Eur. J. Pharmacol. 300: 99-104, 1996.

15. Tortella F.C., Pellicano M. and Bowery N.G., Dextromethorphan and neuromodulation: old drug coughs up new activities. Trends. Pharmacol. Sci. 10: 501-507, 1989.

16. Choi D.W., Dextrorphan and dextromethorphan attenuate glutamate neurotoxicity. Brain Res. 403: 333-336, 1987.

17. Haberecht M.F., Mitchell C.K., Lo G.J. and Redburn D.A., $N$-Methyl-D-aspartate-mediated glutamate toxicity in the developing rabbit retina. J. Neurosci. Res. 47: 416-426, 1997.

18. Liu Y., Qin L., Li G., Zhang W., An L., Liu B. and Hong J.S., Dextromethorphan protects dopaminergic neurons against inflammation-mediated degeneration through inhibition of microglial activation. J. Phannacol. Exp. Ther. 305: 212-218, 2003.

19. Li G., Cui G., Tzeng N.S., Wei S.J., Wang T., Block M.L. and Hong J.S., Femtomolar concentrations of dextromethorphan protect mesencephalic dopaminergic neurons from inflammatory damage. FASEB J. 19: 489-496, 2005.

20. Kim H.C., Nabeshima T., Jhoo W.K., Ko K.H., Kim W.K., Shin E.J., Cho M. and Lee P.H., Anticonvulsant effects of new morphinan derivatives. Bioorg. Med. Chem. Lett. 11: 1651-1654, 2001.
21. Wu C.C., Chiao C.W., Hsiao G., Chen A. and Yen M.H., Melatonin prevents endotoxin induced circulatory failure in rats. J. Pineal Res. 30: 147-156, 2001.

22. Liaw W.J., Tzao C., Wu J.Y., Chen S.J., Wang J.H. and $\mathrm{Wu}$ C.C., Inhibition by terbutaline of nitric oxide and superoxide anion levels of endotoxin-induced organs injury in the anesthetized rat. Shock 19: 281-288, 2003.

23. Hong H.J., Loh S.H. and Yen M.H., Suppression of the development of hypertension by the inhibitor of inducible nitric oxide synthase. Br. J. Pharmacol. 131: 631-637, 2000.

24. Titheradge M.A., Nitric oxide in septic shock. Biochim. Biophys. Acta 1411: 437-455, 1999.

25. Thiemermann C., Nitric oxide and septic shock. Gen. Pharmacol. 29: 159-166, 1997.

26. Ando H., Takamura T., Ota T., Nagai Y. and Kobayashi K., Cerivastatin improves survival of mice with lipopolysaccharide-induced sepsis. J. Pharmacol. Exp. Ther. 294: 1043-1046, 2000.

27. Nathan C., Nitric oxide as a secretory product of mammalian cells. FASEB J. 6: 3051-3064, 1992.

28. Langrehr J.M., Hoffman R.A., Lancaster J.R. Jr. and Simmons R.L., Nitric oxide - a new endogenous immunomodulator. Transplantation 55: 1205-1212, 1993.

29. Szabo C., Wu C.C., Gross S.S., Thiemermann C. and Vane J.R., Interleukin-1 contributes to the induction of nitric oxide synthase by endotoxin in vivo. Eur. J. Pharmacol. 250: $157-160,1993$.

30. Thiemermann C., Wu C.C., Szabo C., Perretti M. and Vane J.R., Role of tumour necrosis factor in the induction of nitric oxide synthase in a rat model of endotoxin shock. Br. J. Pharmacol. 110: 177-182, 1993.

31. Horn K.D., Evolving strategies in the treatment of sepsis and systemic inflammatory response syndrome (SIRS). QJM 91: 265-277, 1998.

32. Kukreja R.C. and Hess M.L., The oxygen free radical system: from equations through membrane protein interactions to cardiovascular injury and protection. Cardiovasc. Res. 26: 641-655, 1992.

33. Wang W., Jittikanont S., Falk S.A., Li P., Feng L., Gengaro P.E., Poole B.D., Bowler R.P., Day B.J., Crapo J.D. and Schrie R.W., Interaction among nitric oxide, reactive oxygen species, and antioxidants during endotoxemia-related acute renal failure. Am. J. Physiol. 284: F532-F537, 2003. 